\title{
Detection of Usutu virus infection in a healthy blood donor from south-west Germany, 2012
}

L Allering ${ }^{1}$, H Jöst ${ }^{1}$, P Emmerich ${ }^{1}$, S Günther ${ }^{1}$, E Lattwein ${ }^{2}$, M Schmidt ${ }^{3}$, E Seifried ${ }^{3}$, V Sambri ${ }^{4}$ K Hourfar ${ }^{3,5}$

J Schmidt-Chanasit (jonassi@gmx.de) ${ }^{1,5}$

1. Bernhard Nocht Institute for Tropical Medicine, WHO Collaborating Centre for Arbovirus and Haemorrhagic Fever Reference and Research, Hamburg, Germany

2. EUROIMMUN Medizinische Labordiagnostika AG, Lübeck, Germany

3. German Red Cross Blood Service Baden-Württemberg-Hesse, Frankfurt, Germany

4. Microbiology Unit, Regional Reference Centre for Microbiological Emergencies, Bologna, Italy

5. The authors contributed equally to this study

Allering L, Jöst H, Emmerich P, Günther S, Lattwein E, Schmidt M, Seifried E, Sambri V, Hourfar K, Schmidt-Chanasit J. Detection of Usutu virus infection in a healthy blood donor from south-west Germany, 2012. Euro Surveill. 2012;17(50):pii=20341. Available online: http://www.eurosurveillance.org/ViewArticle. aspx?Articleld $=20341$

From September 2011 until November 2012, 31 serum samples from German patients with clinically suspected acute Usutu virus (USUV) infections were tested for USUV-specific antibodies. All samples tested negative. In addition, 4,200 serum samples from healthy blood donors from south-west Germany were collected in January 2012 and also analysed for the presence of specific antibodies. One sample tested positive for USUV-IgG and -IgM. Thus, the seroprevalence of USUV antibodies in healthy blood donors from south-west Germany was low in January 2012.

Following the first detection of Usutu virus (USUV) in the city of Weinheim, Germany, in 2010 and subsequent spread of USUV in mosquitoes and birds in south-west Germany 2011 [1], a study was initiated in 2011 to investigate potential human infections. From September 2011 to November 2012, 31 serum samples from patients in Germany with clinically suspected acute Usutu virus (USUV) infections were collected and tested for USUV-specific antibodies. In addition, in January 2012, 4,200 serum samples from healthy blood donors from south-west Germany were collected and also analysed for USUV-specific antibodies. Although all serum samples from the patients tested negative, one IgG- and IgM-positive blood donor was confirmed to have been infected by USUV by virus neutralisation tests, after eliminating the possibility of serological cross-reactivity to other flaviviruses.

\section{Background}

USUV is a mosquito-borne, single-stranded RNA virus that belongs to the Japanese encephalitis virus group within the family Flaviviridae. USUV can cause Usutu fever (USUF) in humans, a mild arboviral disease characterised by fever, rash, jaundice and headache. In contrast, in the immunocompromised patient, USUF can present as acute meningoencephalitis. USUV was originally isolated from Culex neavei mosquitoes in
South Africa in 1959 [2]. In 2001, USUV emerged outside of Africa and caused an epizootic among birds in Austria [3]. In the following years, USUV was found to circulate in several other European countries including Hungary, Switzerland, Spain and Italy [4]. Reports on clinically apparent human USUV infections are scarce and only four cases are described so far in the literature. The first case occurred in 1981 in the Central African Republic and was a patient with fever and rash, and the second case was a 10-year-old patient with fever and jaundice identified in Burkina Faso in 2004 [5]. In 2009, two human cases with an USUV-related neuroinvasive illness were reported from Italy $[6,7]$. Consequently, 359 Italian blood donors were tested for the presence of USUV-specific IgG antibodies [8]. Four healthy blood donors tested positive [8].

In August 2010, USUV strain 1477 was isolated from a pool of Culex pipiens pipiens mosquitoes that were trapped in the city of Weinheim, south-west Germany [9]. In contrast, all mosquitoes trapped during 2009 in the city of Weinheim had tested negative for USUV [9]. After the initial detection in 2010 , the virus spread in 2011 and caused epizootics among wild and captive birds in south-west Germany [1]. We therefore, initiated this study to investigate potential cases of human USUV infection in Germany.

\section{Testing of blood samples for Usutu virus}

From September 2011 until November 2012, 31 blood samples from patients in Germany with clinically suspected acute USUV infections (fever, rash or headache) were sent to the World Health Organization (WHO) Collaborating Centre for Arbovirus and Haemorrhagic Fever Reference and Research at the Bernhard Nocht Institute for Tropical Medicine (BNI) in Hamburg and tested for USUV-specific-IgG and -IgM antibodies with an in-house indirect immunofluorescence assay (IFA). 
The IFA was validated with USUV positive and negative samples from Italy $[6,8]$. All samples tested negative.

In addition 4,200 serum samples from healthy blood donors from south-west Germany were collected in January 2012 and analysed for the presence of USUVspecific-IgG antibodies with the in-house IFA. Serum samples that tested positive were further investigated with a commercial USUV IgG enzyme-linked immunosorbent assay (ELISA) (EUROIMMUN, Lübeck, Germany) and for serological IgG-cross-reactivity to other flaviviruses such as West Nile virus (WNV), tick-borne encephalitis virus (TBEV), Japanese encephalitis virus (JEV) and yellow fever virus (YFV) by endpoint titration in IFA as described recently [10-13]. In addition, samples were further investigated with virus neutralisation tests (VNTs) as previously described $[10,11]$.

Seventy-nine serum samples originating from different healthy blood donors tested USUV-IgG positive in IFA and this result was confirmed by the commercial USUVIgG ELISA. Serological cross-reactivity with TBEV, JEV, WNV and YFV was observed and highest endpoint titres against USUV were only demonstrated for nine samples. For 53 samples, the highest endpoint titres were demonstrated against TBEV, whereas 17 samples showed no titre differences between the analysed flaviviruses. As VNT is considered the gold standard for flavivirus serology, the 79 IFA-reactive samples were tested against USUV, WNV, JEV, TBEV and YFV by VNT. One sample showed neutralising antibodies against WNV and for 77 samples highest neutralising antibody titres were demonstrated against TBEV. Only one sample showed a neutralising antibody titre against USUV.

\section{Further investigation of the positive blood sample}

Interestingly, the sample with a neutralising antibody titre against USUV also tested positive for USUV- and WNV-IgM in the in-house IFA (Table), demonstrating recent USUV infection. Consequently, four blood samples of the USUV-IgG and IgM positive tested blood donor, taken in the years 2007, 2008, 2010 and 2011 were provided to the BNI for further analysis. An USUVIgG- and -IgM seroconversion was demonstrated in the blood sample from the year 2011 when compared to the sample from the year 2010 (Table). Moreover, a low anti-TBEV-IgG titre of 1:80 and 1:160 was detected in samples from the years 2008 and 2010, respectively (Table). This titre is most probably related to a previous TBEV vaccination. In addition, one sample showed the highest neutralising antibody titre against WNV (data not shown) and tested negative for WNV-IgM in the inhouse IFA, demonstrating a past WNV infection.

The blood donor testing positive for USUV-IgG and -IgM, who was in his forties, reported no history of vaccination against YFV and JEV, and did not have a fever during a period of three months before the blood donation. In addition, he had not been abroad during this period. The blood donor lives in the city of GrossGerau (Figure) and dead black birds testing positive for USUV were reported from the district of Gross-Gerau in 2011 [1]. These findings, taken together with the serological results corroborate the hypothesis of a paucior asymptomatic and autochthonous USUV infection of the blood donor during late summer 2011.

TABLE

Results of serological analysis of a German blood donor at different time points, Germany, 2007-2012

\begin{tabular}{|c|c|c|c|c|c|c|c|c|c|c|c|c|c|c|}
\hline \multirow{3}{*}{ Virus } & \multicolumn{12}{|c|}{ Immunofluorescence assay (IFA) } & \multicolumn{2}{|c|}{$\begin{array}{c}\text { Virus } \\
\text { neutralisation } \\
\text { test (VNT) }\end{array}$} \\
\hline & \multicolumn{2}{|c|}{ April 2007} & \multicolumn{2}{|c|}{ October 2008} & \multicolumn{2}{|c|}{ October 2010} & \multicolumn{2}{|c|}{ October 2011} & \multicolumn{2}{|c|}{ January 2012} & \multicolumn{2}{|c|}{ April 2012} & \multirow{2}{*}{$\begin{array}{c}\text { January } \\
2012\end{array}$} & \multirow{2}{*}{$\begin{array}{l}\text { April } \\
2012\end{array}$} \\
\hline & $\lg G$ & $\lg M$ & $\lg G$ & $\lg M$ & $\lg G$ & $\lg M$ & $\lg G$ & $\lg M$ & $\lg G$ & $\lg M$ & $\lg G$ & $\lg M$ & & \\
\hline Usutu & Neg & Neg & Neg & Neg & Neg & $\mathrm{Neg}$ & $1: 1,280$ & $1: 160$ & $1: 2,560$ & $1: 160$ & $1: 2,560$ & $1: 40$ & $1: 40$ & $1: 80$ \\
\hline West Nile & Neg & $\mathrm{Neg}$ & Neg & Neg & Neg & Neg & $1: 640$ & $1: 20$ & $1: 640$ & $1: 20$ & $1: 160$ & $1: 20$ & $\mathrm{Neg}$ & $\mathrm{Neg}$ \\
\hline Japanese encephalitis & $\mathrm{Neg}$ & Neg & Neg & $\mathrm{Neg}$ & Neg & Neg & $1: 2,560$ & Neg & $1: 1,280$ & Neg & $1: 640$ & Neg & Neg & $\mathrm{Neg}$ \\
\hline Tick-borne encephalitis & $\mathrm{Neg}$ & Neg & 1:80 & $\mathrm{Neg}$ & $1: 160$ & $\mathrm{Neg}$ & $1: 1,280$ & Neg & $1: 1,280$ & $\mathrm{Neg}$ & $1: 1,280$ & Neg & Neg & $1: 20$ \\
\hline Yellow fever & Neg & $\mathrm{Neg}$ & Neg & Neg & $1: 160$ & $\mathrm{Neg}$ & $1: 1,280$ & Neg & $1: 1,280$ & Neg & $1: 160$ & Neg & Neg & Neg \\
\hline
\end{tabular}

Neg: negative.

Immunofluorescence assay and virus neutralisation test results of $<1: 20$ were considered negative. 


\section{FIGURE}

Origin of samples from healthy blood donors, Germany, January $2012(\mathrm{n}=4,200)$

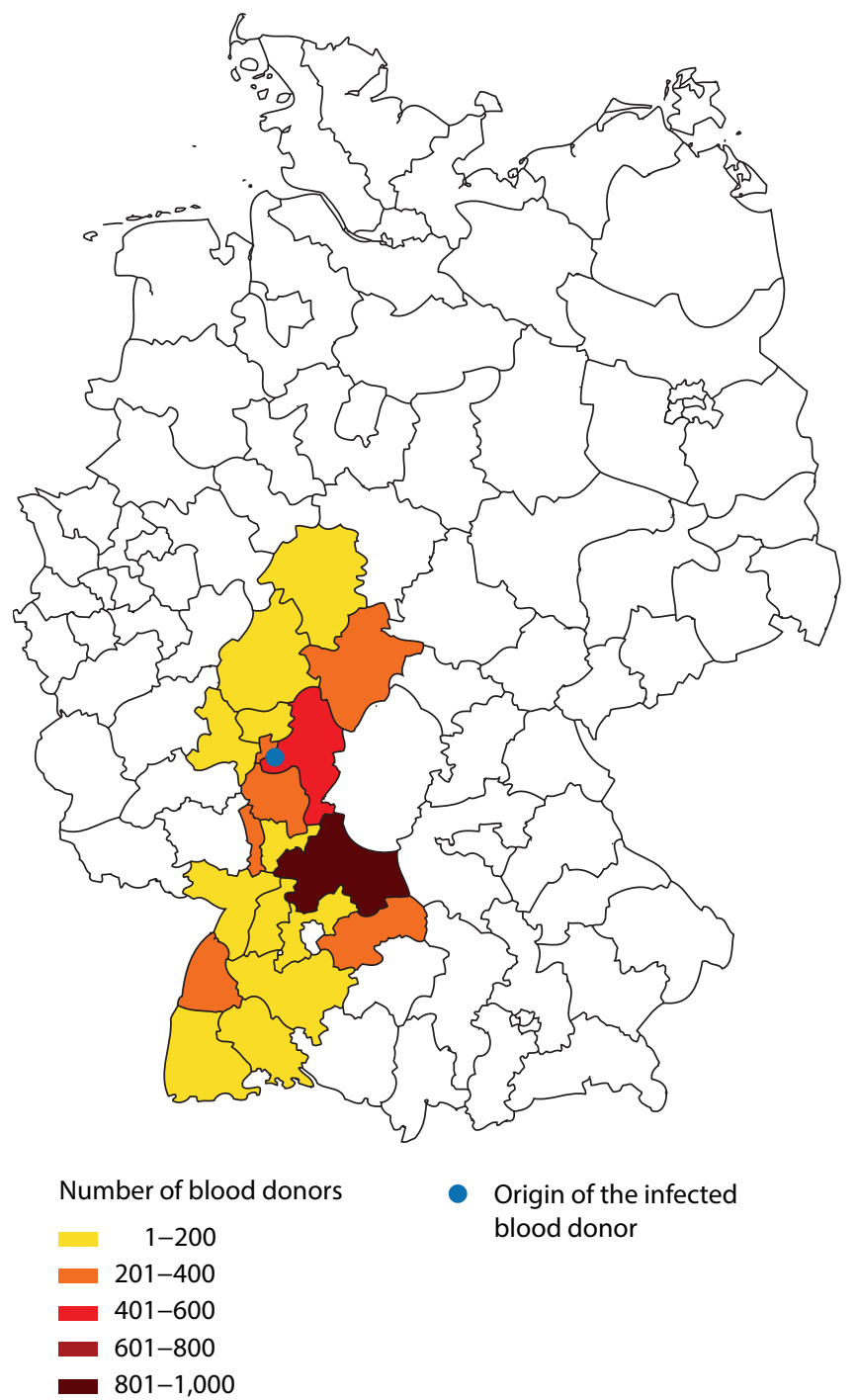

The origin of blood donor serum samples is according to postal code regions. The blue dot represents the city of Gro $\beta-G e r a u$, origin of the blood donor sample testing positive for Usutu virus-IgG and -IgM.

\section{Conclusions}

In Italy in 2011, it was shown, that USUV infection can induce clinically asymptomatic viraemic episodes in humans [14]. This, together with the facts that USUV can cause severe disease in immunocompromised patients and could be a potential emerging vectorborne disease in Europe, highlights the need for surveillance to provide information necessary to take adequate prevention measures early. Thus, it was suggested that active human surveillance should be implemented in Europe and performed by detection of USUV RNA in blood donor samples [4]. However, an additional screening of organ donors for USUV IgG and USUV RNA could become relevant in south-west Germany during late summer. Screening for USUV RNA in cerebrospinal fluid samples from acute meningoencephalitis cases should be performed as well [4]. In conclusion, public health authorities, blood transfusion services and clinicians in Germany should be aware of the risk of USUV infection in humans, especially during late summer.

\section{References}

1. Becker N, Jöst H, Ziegler U, Eiden M, Höper D, Emmerich P, et al. Epizootic emergence of Usutu virus in wild and captive birds in Germany. PLoS One. 2012;7(2):e32604.

2. Adam F, Digoutte JP. Virus d'Afrique (Base de Données). Centre Collaborateur OMS de Référence et de Recherche pour les Arbovirus et les Virus de Fièvres Hémorrhagiques (CRORA) [African viruses (database). WHO Collaborating Centre for Arbovirus and Haemorrhagic Fever Reference and Research]. Dakar: Institut Pasteur de Dakar. [Accessed 21 Sep 2012]. French. Available from: http://www.pasteur.fr/recherche/ banques/CRORA/

3. Weissenböck H, Kolodziejek J, Url A, Lussy H, Rebel-Bauder B, Nowotny N. Emergence of Usutu virus, an African mosquitoborne flavivirus of the Japanese encephalitis virus group, central Europe. Emerg Infect Dis. 2002;8(7):652-6.

4. Vazquez A, Jimenez-Clavero M, Franco L, Donoso-Mantke O, Sambri V, Niedrig M, et al. Usutu virus: potential risk of human disease in Europe. Euro Surveill. 2011;16(31): pii=19935. Available from: http://www.eurosurveillance.org/ViewArticle. aspx?Articleld $=19935$

5. Nikolay B, Diallo M, Boye CS, Sall AA. Usutu virus in Africa. Vector Borne Zoonotic Dis. 2011;11(11):1417-23.

6. Cavrini F, Gaibani P, Longo G, Pierro AM, Rossini G, Bonilauri $P$, et al. Usutu virus infection in a patient who underwent orthotropic liver transplantation, Italy, August-September 2009. Euro Surveill. 2009;14(50):pii=19448. Available from: http://www.eurosurveillance.org/ViewArticle. aspx?Articleld $=19448$

7. Pecorari M, Longo G, Gennari W, Grottola A, Sabbatini AM, Tagliazucchi S, et al. First human case of Usutu virus neuroinvasive infection, Italy, August-September 2009. Euro Surveill. 2009;14(50):pii=19446. Available from: http://www. eurosurveillance.org/ViewArticle.aspx?Articleld=19446

8. Gaibani P, Pierro A, Alicino R, Rossini G, Cavrini F, Landini MP, et al. Detection of Usutu-virus-specific IgG in blood donors from northern Italy. Vector Borne Zoonotic Dis. 2012;12(5):431-3.

9. Jöst H, Bialonski A, Maus D, Sambri V, Eiden M, Groschup MH, et al. Isolation of Usutu virus in Germany. Am J Trop Med Hyg. 2011;85(3):551-3.

10. Schultze-Amberger J, Emmerich P, Günther S, Schmidt-Chanasit J. West Nile virus meningoencephalitis imported into Germany. Emerg Infect Dis. 2012;18(10):1698-700.

11. Tappe D, Schmidt-Chanasit J, Ries A, Ziegler U, Müller A, Stich $A$. Ross River virus infection in a traveller returning from northern Australia. Med Microbiol Immunol. 2009;198(4):271-3.

12. Tappe D, Nemecek A, Zipp F, Emmerich P, Gabriel M, Günther S, et al. Two laboratory-confirmed cases of Japanese encephalitis imported to Germany by travelers returning from Southeast Asia. J Clin Virol. 2012;54(3):282-5.

13. Jöst H, Bürck-Kammerer S, Hütter G, Lattwein $\mathrm{E}$, Lederer $\mathrm{S}$, Litzba N, et al. Medical importance of Sindbis virus in southwest Germany. J Clin Virol. 2011;52(3):278-9.

14. Gaibani P, Pierro AM, Cavrini F, Rossini G, Landini MP, Sambri V. False positive transcription-mediated amplification assay detection of West Nile virus in blood from a patient with viremia caused by an Usutu virus infection. J Clin Microbiol. 2010;48(9):3338-9. 